Published in final edited form as:

Angew Chem Int Ed Engl. 2014 March 24; 53(13): 3362-3366. doi:10.1002/anie.201308368.

\title{
Hypoxia-targeted siRNA delivery
}

\author{
F Perche ${ }^{\dagger}$, \\ Department of Pharmaceutical Sciences, Bouve College of Health Sciences, Center for \\ Pharmaceutical Biotechnology and Nanomedicine, Northeastern University, 140 The Fenway, \\ Room 230, 360 Huntington Avenue, Boston, MA 02115 (USA)
}

\section{S Biswas ${ }^{\dagger}$,}

Birla Institute of Technology \& Science-Pilani, Hyderabad Campus, Jawahar Nagar, Shameerpet Mandal, A.P - 500078, India

\section{T Wang,}

Department of Pharmaceutical Sciences, Bouve College of Health Sciences, Center for Pharmaceutical Biotechnology and Nanomedicine, Northeastern University, 140 The Fenway, Room 230, 360 Huntington Avenue, Boston, MA 02115 (USA)

\section{Zhu, and}

Department of Pharmaceutical Sciences, Bouve College of Health Sciences, Center for Pharmaceutical Biotechnology and Nanomedicine, Northeastern University, 140 The Fenway, Room 230, 360 Huntington Avenue, Boston, MA 02115 (USA)

Irma Lerma Rangel College of Pharmacy, Texas A\&M University Health Science Center, Kingsville, Texas 78363 (USA)

\section{VP Torchilin ${ }^{*}$}

Department of Pharmaceutical Sciences, Bouve College of Health Sciences, Center for Pharmaceutical Biotechnology and Nanomedicine, Northeastern University, 140 The Fenway, Room 230, 360 Huntington Avenue, Boston, MA 02115 (USA)

\section{Keywords}

cancer; antitumor agents; siRNA delivery; tumor targeting; hypoxia-triggered copolymer

\begin{abstract}
Altered vasculature and resultant chaotic tumor blood flow lead to the appearance in fastgrowing tumors of the regions with gradients of oxygen tension and acute hypoxia (less than $1.4 \%$ oxygen). ${ }^{[1]}$ Due to its roles in tumorigenesis and resistance to therapy, hypoxia represents problem in cancer therapy. ${ }^{[1-2]}$ Insufficient therapeutic agent delivery to the hypoxic regions in solid tumors is recognized as one of the causes of resistance to therapy. ${ }^{[1,3]}$ This led to the development of hypoxia imaging agents ${ }^{[4]}$, and the use of
\end{abstract}

\footnotetext{
*Fax: (+1 +1 617373 8886), v.torchilin@neu.edu.

†both authors contributed equally and should both be considered first authors.

Author contributions

F.P. and S.B. designed and carried out the experiments. V.P.T. supervised the project. F.P, S.B. and V.P.T. analysed the data and wrote the manuscript. L. Z. gave technical support and conceptual advice. T. W. performed the exvivo imaging experiment.
} 
hypoxia-activated anticancer prodrugs. ${ }^{[2 \mathrm{a}]}$ Here we show the first example of the hypoxiainduced siRNA uptake and silencing using a polyethylene glycol 2000-azobenzenepolyethyleneimine $1.8 \mathrm{kDa}$-1,2-dioleyl-sn-glycero-3-phosphoethanolamine (PAPD)-based nanocarrier where azobenzene imparts hypoxia sensitivity and specificity. ${ }^{[4 a]}$ We report hypoxia-activated green fluorescent protein (GFP) silencing in vitro and its downregulation in GFP expressing tumors after intravenous administration. The proposed nanoformulation represents a novel tumor environment-responsive modality for cancer targeting and siRNA delivery.

The specificity and potency of siRNA regulation of gene expression holds great promise for cancer therapy. ${ }^{[5]}$ However, siRNA delivery to hypoxic regions is challenging since such regions are distant from blood vessels and have increased efflux transporters. ${ }^{[1]}$ In addition, the use of nanocarriers is required to protect siRNA from degradation and to promote its cellular internalization and endosomal escape. ${ }^{[5 a]}$ Usually, nanoparticle uses rely on the enhanced permeability and retention (EPR) effect for tumor accumulation. ${ }^{[6]}$ Nanoparticles are expected to show preferential extravasation from the circulation when reaching the altered tumor vasculature with its widened endothelial fenestrae and deficient pericyte coverage. Conjugation of PEG to nanoparticles extends their blood circulation time, increasing the probability of tumor accumulation by EPR. ${ }^{[7]}$ However, PEGylation can also hinder cellular uptake resulting in decreased therapeutic activity. ${ }^{[5 a, 7 a]}$ This PEG dilemma led to the design of nanoformulations with tumor-stimuli detachable PEG to target payload delivery. ${ }^{[6,8]}$ Nitroimidazole derivatives have been proposed as hypoxia-sensors since they are subject to intracellular reduction with formation of free radicals. ${ }^{[1 a, 2 b, 4 b]}$ While this free radicals rapidly oxidize by molecular oxygen, their stabilization under hypoxia leads to reduction-mediated cleavage. ${ }^{[1 \mathrm{~b}, 4 \mathrm{a}, 4 \mathrm{~b}, 9]}$ Nagano and coworkers demonstrated successful hypoxia imaging in vivo with azobenzene-based probes. ${ }^{[4 a, 9]}$ In our study, we used azobenzene as hypoxia-responsive bioreductive linker for hypoxia-targeted siRNA delivery with PEGylated nanopreparations upon PEG de-shielding. The production of GFP was used as a model of siRNA downregulation in both in vitro and in vivo studies.

The potency of that azobenzene bond for siRNA delivery was evaluated by linking azobenzene to PEG2000 at one end and to PEI $1.8 \mathrm{kDa}$-DOPE conjugate on the other to obtain PAPD (Figure 1A).

PEG2000 was used as the hydrophilic block and for imparting stability in circulation. [8b, 10] The PEI-DOPE conjugate, was introduced for siRNA complexation and to promote formation of micellar nanoparticles. ${ }^{[11]}$ The hypoxia-sensitive polymer PAPD and its nonsensitive PEG-PEI-DOPE (PPD) counterpart were synthetized (Figs. S1-S6) and expected to condense siRNA into nanoparticles with a PEG layer to protect it from the nuclease attack and impart stability in physiological fluids (Fig. 1B). ${ }^{[7,}$, 8d, 10] The PEG groups would be detached from PAPD/siRNA complexes in the hypoxic and reductive ${ }^{[1 b, 12]}$ tumor environment because of azobenzene linker degradation, leading to exposure of PEI's positive charge to promote cellular internalization of remaining PEI-DOPE/siRNA complexes. ${ }^{[2 \mathrm{c}, 8 \mathrm{e}, 11 \mathrm{~b}]}$ 
Formation of complexes between PAPD and siRNA was demonstrated by EtBr exclusion and transmission electron microscopy (Figures 2A, 2D). In line with previous results, ${ }^{[13]} \mathrm{a}$ higher N/P ratio of PAPD over PEI was required to quench siRNA fluorescence ( 16 and 4 , respectively). Complexes protected siRNA against RNAse degradation (Figure 2B), demonstrated moderate unpacking (30\% increase in EtBr fluorescence, Figure 2C) after incubation in the medium containing $10 \%$ foetal bovine serum, in agreement with. ${ }^{[7 b, 8 e, 13-14]}$

Since reductases-rich rat liver microsomes were reported to cleave nitroimidazole derivatives under hypoxia, $\left.{ }^{[4 a, 4 b}, 9\right]$ we evaluated siRNA condensation and uptake of the complexes after incubation with rat liver microsomes (Figures 2C, 3). While siRNA fluorescence was quenched in PBS (26\% of siRNA fluorescence), the incubation with microsomes led to the 3-fold fluorescence increase (Figure 2C) and 3-fold increase in aniline absorbance (Figure S8), supporting bioreductive cleavage. ${ }^{[4 \mathrm{~b}, 12]}$ Addition of microsomes also led to a considerable positive charge increase from $0.1 \pm 6.5 \mathrm{mV}$ to $13.2 \pm 3.7 \mathrm{mV}(\mathrm{p}=$ 0.006 , Student's $t$ test) (Figures 2E, 2F). Exposure of positive surface charges from the siRNA complexes which were previously hidden by PEG under reductive hypoxia conditions indicates PEG detachment after azobenzene cleavage. ${ }^{[2 c, 4 a, 8 a]}$ By contrast, no such charge exposure was observed for PPD/siRNA complexes (Figure S9). No cytotoxicity was detected after the treatment with PPD and PAPD both free and complexed with siRNA and both in normoxic and hypoxic conditions (Figures S10, S11).

We performed uptake studies of nanopreparations by cancer cells monolayer cultures in normoxic and hypoxic environment. In vitro hypoxia was confirmed by Hydroxyprobe staining (Figure S12) ${ }^{[4 \mathrm{c}]}$. Cellular internalization of PPD or PAPD complexes prepared with FAM-labelled siRNA was determined by flow cytometry (Figures 3A, B). The cellassociated fluorescence of PAPD/siRNA-treated cells under hypoxia was 3.2-fold higher than under normoxia (13.4 and 4.1, respectively; Figure 3B) and 3.9-fold higher than for $\mathrm{PPD} /$ siRNA under hypoxia.

Cancer cell spheroids have been proposed as models for evaluation of nanomedicines, ${ }^{[15]}$ and we used a spheroid model to confirm hypoxia-activated siRNA internalization. Whereas free FAM-siRNA fluorescence was bound to the surface of spheroids, complexation with PAPD or PPD nanocarriers increased its penetration under normoxia, although only to the first cell layers (Figures 3, S13), as reported by Wong et al with siRNA lipoplexes. ${ }^{[16]}$ Only with treatment of spheroids with PAPD/siRNA under hypoxia (Figures 3E, S13) resulted in further increase of siRNA penetration. This was matched with a deeper penetration of rhodamine-labeled PEG-Azo-Rhodamine-PEI-DOPE (PARPD) over PEG-Rhodamine-PEIDOPE (PRPD) (Figure S14). Altogether, this data suggests better uptake of the nanoformulation after PEG deshielding. ${ }^{[2 c, 8 a, 8 d, 8 e]}$

We used HeLa cells stably expressing GFP (HeLa/GFP) and some other cells to confirm the PAPD-mediated gene down-regulation in the presence of 10\% FBS (Figures 4, S15).Whereas no GFP down-regulation was observed with PAPD-complexed siRNA under normoxia (Figure 4A), a 30-40\% down-regulation was detected under hypoxia, in all HeLa/ GFP, NCI-ADR-RES/GFP and A2780/GFP cells (Figures 4A, S16) without significant 
down-regulation when using insensitive PPD/siRNA polyplexes (Figure 4B). This silencing activity is comparable with reported earlier using $200 \mathrm{nM}$ siRNA. ${ }^{[16]}$ Hypoxia-induced silencing was concordant with the internalization results (Figure 3). The moderate silencing activity observed in vitro compared to Lipofectamine-mediated delivery may be attributed to incomplete cleavage of the azobenzene bond within the observation time, however one has to note that the silencing of Lipofectamine complexes is identical under both, normoxia and hypoxia, which clearly supports the hypoxic selectivity of our nanocarrier. To corroborate down-regulation in hypoxic conditions with internalization, we formed polyplexes using rhodamine B-labeled copolymers (Figures $4 \mathrm{C}-\mathrm{F}$ ). Stronger GFP down-regulation under hypoxia over normoxia was proportional to the enhanced PARPD uptake (Figures 4C, D) while an opposite correlation was observed for PRPD (Figures 4D-F).

We then performed a tumor accumulation study of the copolymers $4 \mathrm{~h}$ after intravenous administration of PARPD and PRPD to mice bearing hypoxic B16F10 tumors ${ }^{[3]}$ (Figures S12, S17-19). Two-fold increased tumor cell-associated fluorescence intensity was observed only for PARPD (Figure S17). Fluorescence from polymers was also detected in the blood filtering organs liver, spleen and kidney (Figures S17, S18), off-target sites for nanomedicines. ${ }^{[2 c, 5 a]}$ Whereas PARPD was detected in tumor sections, almost no tumor accumulation was detected for PRPD. The data support tumor hypoxia-induced PEG shedding with subsequent PARPD uptake upon the charge exposure, in good agreement with reports on tumor-specific charge exposure. ${ }^{[2 c, 8 \mathrm{a}, 8 \mathrm{~d}, 8 \mathrm{e}]}$

We finally assessed the ability of GFP silencing in vivo on A2780/GFP tumors in mice (Figure 5). Substantial GFP down-regulation was detected after intravenous injection of PAPD/siRNA nanoparticles both by ex vivo imaging (Figure 5A, 24\%), and flow cytometry (Figure 5B, 32\%). No down-regulation was observed with PAPD complexes formed with scramble siRNA (Figure 5). The in vivo silencing capacity of PAPD in vivo corresponded well to its in vitro uptake and silencing profiles (Figures 3, 4, S15) and tumor accumulation (Figures S17, S18).

The PAPD nanocarrier possesses the following properties: a) PEG for stability in physiological fluids and increased tumor accumulation ${ }^{[6,10]}$ (Figures 2, S17), b) PEI-DOPE for siRNA complexation, protection form RNAse degradation and endosomal escape $^{[8 \mathrm{e}, 11 \mathrm{~b}, 11 \mathrm{c}]}$ (Figure 2), c) Azobenzene linker for hypoxia-activated charge exposure $^{[2 b, 4 b, 8 a, 11 a, 13,16]}$ (Figures 2, S8) to allow d) Hypoxia-dependent cellular uptake and tumor accumulation of the copolymer (Figures 5, S18) and GFP silencing in vivo after intravenous administration (Figure 5) without detectable cytotoxicity (Figures S10, S11, S20).

Few examples of hypoxia-targeted delivery have been reported. ${ }^{[2 b, 2 c]}$ Our study is, to the best of our knowledge, the first of its kind with hypoxia-activated siRNA nanocarrier achieving silencing in vivo. The low oxygen threshold (0.1-1\%) of the azobenzene-based probe described by Kiyose et al. ${ }^{[4 a]}$ suggests biosafety of PAPD since side-effects of tirapazamine were attributed to its high hypoxic activation threshold. ${ }^{[2 \mathrm{a}]}$ However, we only detected a moderate silencing activity in vitro and in vivo indicating the requirement for optimization of the structure. We suggest two axes for further improvement. On the one 
hand, a compromise between tumor hypoxia-activation and cellular uptake by optimization of the chemical structure as in Piao et al. ${ }^{[9]}$ On the other hand, mixed micelles of PAPD and another copolymer could be prepared for improved silencing activity. ${ }^{[17]}$ Finally, since hypoxia is one of the characteristics of drug-resistant cancers, ${ }^{[1]}$ hypoxia-targeted codelivery of a chemotherapeutic drug and siRNA against the hypoxia principal effector, hypoxia inducible factor $1 a$, could allow relapse-free destruction of some of the most aggressive tumors.

\section{Supplementary Material}

Refer to Web version on PubMed Central for supplementary material.

\section{Acknowledgments}

This work was supported by grant U54CA151881 to Dr. Torchilin. We are grateful to Professor Amiji for providing access to the Kodak Imager and William Fowle for transmission electron microscopy.

\section{References}

1. a) Brown JM, Wilson WR. Nat. Rev. Cancer. 2004; 4:437-447. [PubMed: 15170446] b) Wilson WR, Hay MP. Nat. Rev. Cancer. 2011; 11:393-410. [PubMed: 21606941]

2. a) Kling J. Nat. Biotechnol. 2012; 30:381. [PubMed: 22565952] b) Lin Q, Bao C, Yang Y, Liang Q, Zhang D, Cheng S, Zhu L. Adv. Mater. 2013; 25:1981-1986. [PubMed: 23401259] c) Poon Z, Chang D, Zhao X, Hammond PT. ACS Nano. 2011; 5:4284-4292. [PubMed: 21513353]

3. Kieda C, El Hafny-Rahbi B, Collet G, Lamerant-Fayel N, Grillon C, Guichard A, Dulak J, Jozkowicz A, Kotlinowski J, Fylaktakidou KC, Vidal A, Auzeloux P, Miot-Noirault E, Beloeil JC, Lehn JM, Nicolau C. J Mol Med (Berl). 2013; 91:883-899. [PubMed: 23471434]

4. a) Kiyose K, Hanaoka K, Oushiki D, Nakamura T, Kajimura M, Suematsu M, Nishimatsu H, Yamane T, Terai T, Hirata Y, Nagano T. J. Am. Chem. Soc. 2010; 132:15846-15848. [PubMed: 20979363] b) Kizaka-Kondoh S, Konse-Nagasawa H. Cancer Sci. 2009; 100:1366-1373. [PubMed: 19459851] c) Krohn KA, Link JM, Mason RP. J. Nucl. Med. 2008; 49(Suppl. 2):129S-148S. [PubMed: 18523070]

5. a) Huang L, Liu Y. Annu. Rev. Biomed. Eng. 2011; 13:507-530. [PubMed: 21639780] b) Kim DH, Rossi JJ. Nat. Rev. Genet. 2007; 8:173-184. [PubMed: 17304245] c) Weiss GJ, Chao J, Neidhart JD, Ramanathan RK, Bassett D, Neidhart JA, Choi CH, Chow W, Chung V, Forman SJ, Garmey E, Hwang J, Kalinoski DL, Koczywas M, Longmate J, Melton RJ, Morgan R, Oliver J, Peterkin JJ, Ryan JL, Schluep T, Synold TW, Twardowski P, Davis ME, Yen Y. Invest. New Drugs. 2013; 31:986-1000. [PubMed: 23397498]

6. Maeda H, Nakamura H, Fang J. Adv. Drug Deliv. Rev. 2013; 65:71-79. [PubMed: 23088862]

7. a) Bae YH, Park K. J. Control. Release. 2011; 153:198-205. [PubMed: 21663778] b) Ogris M, Brunner S, Schuller S, Kircheis R, Wagner E. Gene Ther. 1999; 6:595-605. [PubMed: 10476219]

8. a) Li JJ, Ge ZS, Liu SY. Chem. Comm. 2013; 49:6974-6976. [PubMed: 23802223] b) Hatakeyama H, Akita H, Kogure K, Oishi M, Nagasaki Y, Kihira Y, Ueno M, Kobayashi H, Kikuchi H, Harashima H. Gene Ther. 2007; 14:68-77. [PubMed: 16915290] c) Ishida T, Kirchmeier MJ, Moase EH, Zalipsky S, Allen TM. Biochim. Biophys. Acta. 2001; 1515:144-158. [PubMed: 11718670] d) Takae S, Miyata K, Oba M, Ishii T, Nishiyama N, Itaka K, Yamasaki Y, Koyama H, Kataoka K. J. Am. Chem. Soc. 2008; 130:6001-6009. [PubMed: 18396871] e) Yang XZ, Du JZ, Dou S, Mao CQ, Long HY, Wang J. ACS Nano. 2012; 6:771-781. [PubMed: 22136582]

9. Piao W, Tsuda S, Tanaka Y, Maeda S, Liu F, Takahashi S, Kushida Y, Komatsu T, Ueno T, Terai T, Nakazawa T, Uchiyama M, Morokuma K, Nagano T, Hanaoka K. Angew. Chem. Int. Ed. Engl. 2013; 52:13028-13032. [PubMed: 24127124]

10. Klibanov AL, Maruyama K, Beckerleg AM, Torchilin VP, Huang L. Biochim. Biophys. Acta. 1991; 1062:142-148. [PubMed: 2004104] 
11. a) Navarro G, Sawant RR, Biswas S, Essex S, Tros de Ilarduya C, Torchilin VP. Nanomedicine (Lond). 2012; 7:65-78. [PubMed: 22191778] b) Sawant RR, Sriraman SK, Navarro G, Biswas S, Dalvi RA, Torchilin VP. Biomaterials. 2012; 33:3942-3951. [PubMed: 22365809] c) Navarro G, Essex S, Sawant RR, Biswas S, Nagesha D, Sridhar S, de ILarduya CT, Torchilin VP. Nanomedicine: Nanotechnology, Biology and Medicine. 2013

12. JooáJung H, GeoláLee T, DongáChung T. Chem. Comm. 2010; 46:3863-3865. [PubMed: 20442912]

13. Malek A, Czubayko F, Aigner A. J. Drug Target. 2008; 16:124-139. [PubMed: 18274933]

14. Burke RS, Pun SH. Bioconjug. Chem. 2008; 19:693-704. [PubMed: 18293906]

15. Mehta G, Hsiao AY, Ingram M, Luker GD, Takayama S. J. of Control. Release. 2012; 164:192204. [PubMed: 22613880]

16. Wong HL, Shen Z, Lu Z, Wientjes MG, Au JL. Mol. Pharm. 2011; 8:833-840. [PubMed: 21417439]

17. Omedes Pujol M, Coleman DJ, Allen CD, Heidenreich O, Fulton DA. J. Control. Release. 2013; 172:939-945. [PubMed: 24140749] 
a
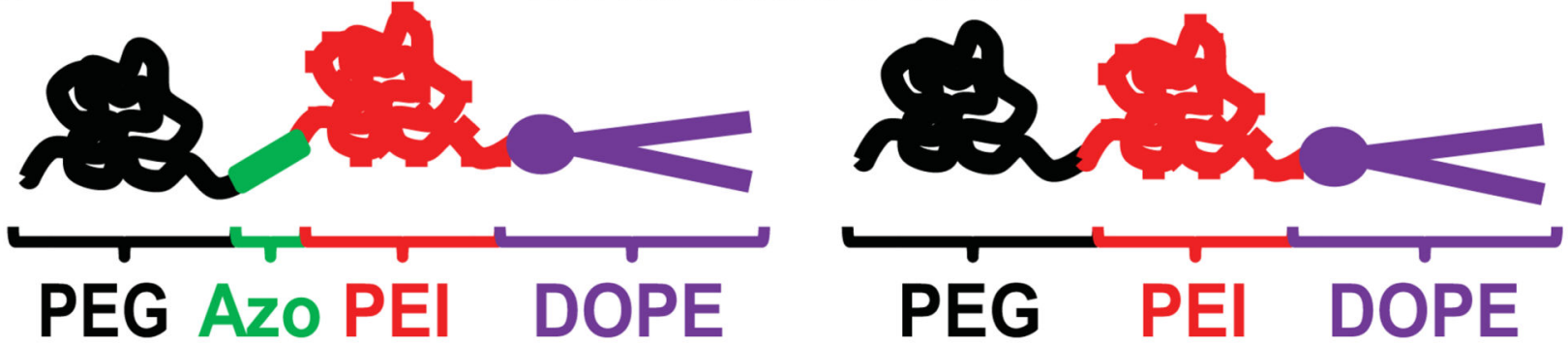

b
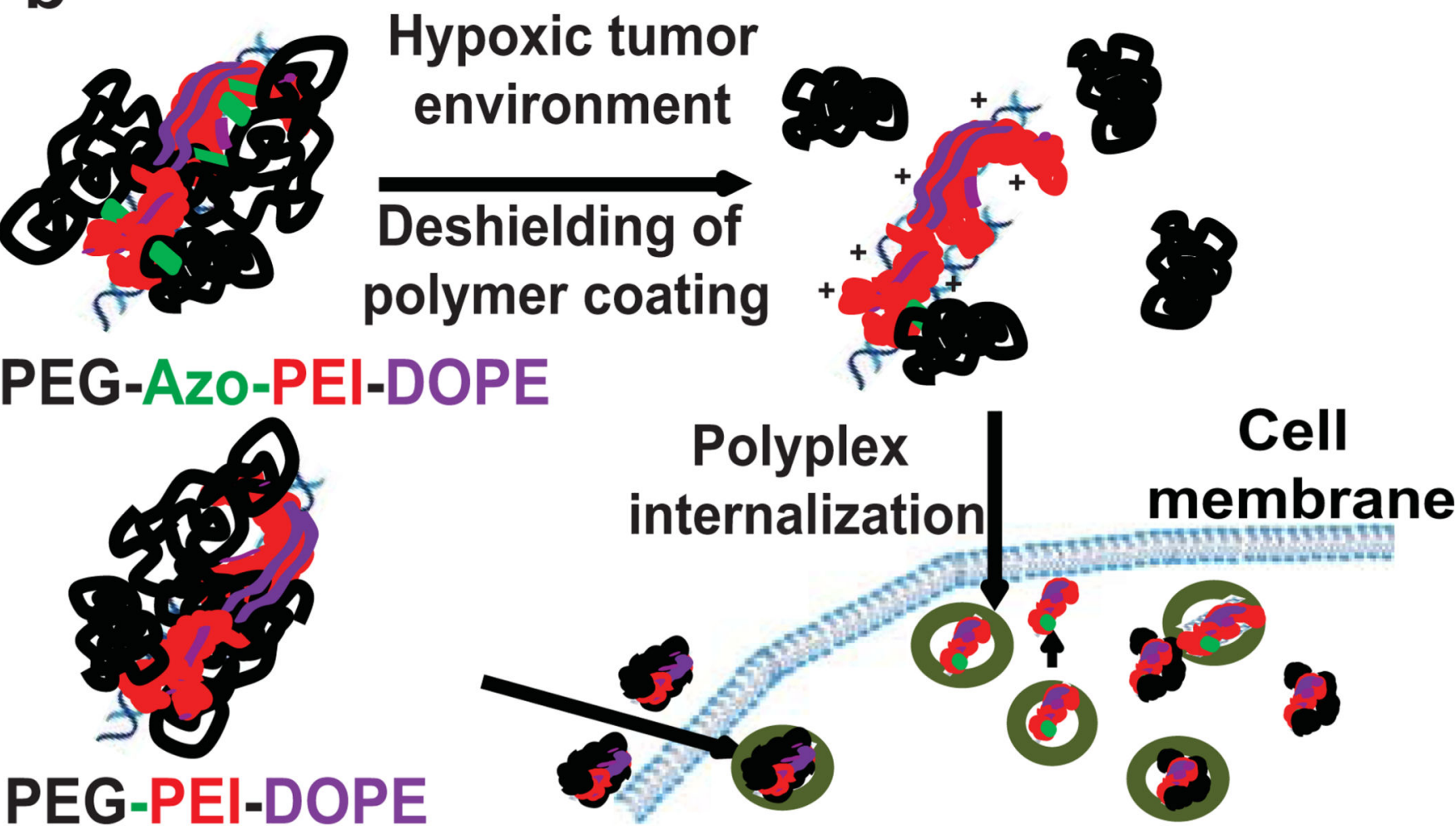

Enhanced celular uptake for stimuli-sensitive system due to hypoxia-induced deshielding

Figure 1.

A) Schematic representation of the synthesized polymers and B) proposed mechanism of internalization in hypoxic tumor micro-environment. 

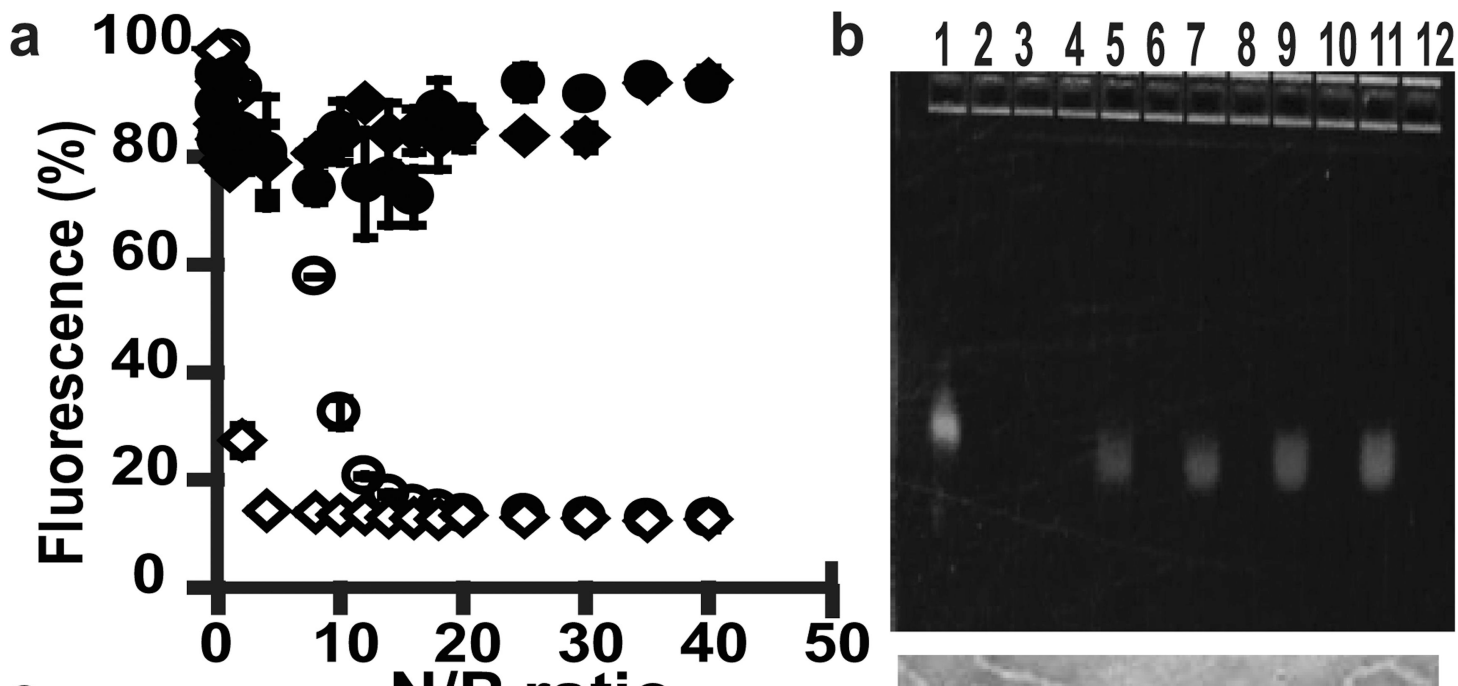

C N/P ratio
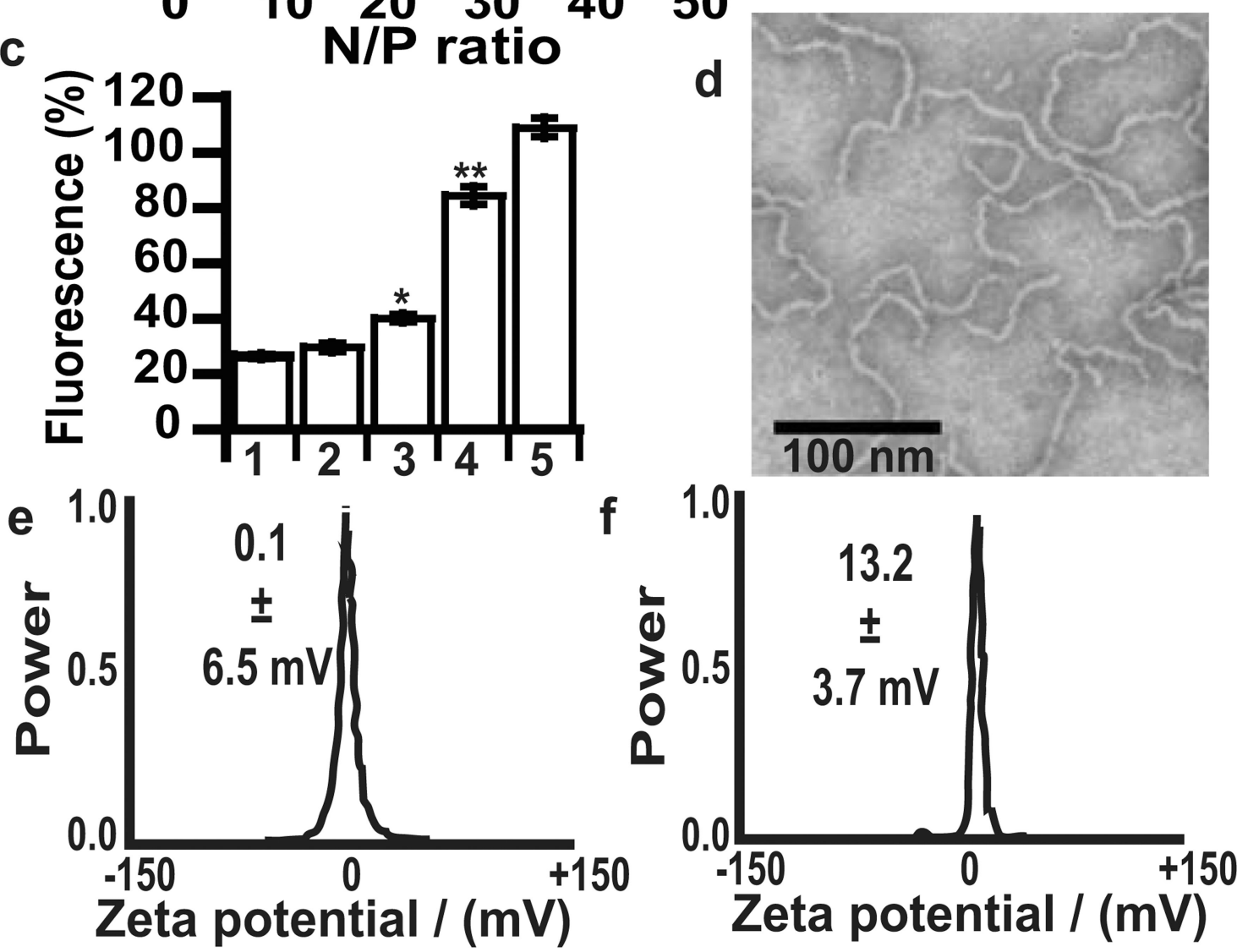

Figure 2. siRNA binding and cytotoxicity

A) Ethidium bromide exclusion assay: $\diamond$, PEI $1.8 \mathrm{kDa}$; $\bigcirc$, PEG-Azo-PEI-DOPE (PAPD), $\diamond$, PEI 1.8 kDa polyplexes treated with Heparin; $\bigcirc$, PEG-Azo-PEI-DOPE polyplexes treated with heparin; B) RNAse protection assay. 1, untreated free siRNA; 2, RNAse-treated free siRNA; 4, PAPD polyplexes, N/P 40; 5, PAPD polyplexes, N/P 40 treated with RNAse and heparin; 6, PAPD polyplexes, N/P 60; 7, PAPD polyplexes, N/P 60 treated with RNAse and heparin; 8, PEG-PEI-DOPE (PPD) polyplexes, N/P 40; 9, PPD polyplexes, N/P 40 treated with RNAse and heparin; 10, PPD polyplexes, N/P 60; 11, PPD polyplexes, N/P 60 
treated with RNAse and heparin, wells 3 and 12 were unused; C) siRNA signal from PAPD polyplexes prepared at an N/P ratio of 40 and incubated $2 \mathrm{~h}$ in 1: PBS; 2: 0\% FBS media; 3 : 10\% FBS N2-bubbled media; 4: 10\% FBS N $\mathrm{N}_{2}$-bubled media and microsomes; 5: PBS followed by heparin treatment, ${ }^{*} \mathrm{p}<0.05$, ** $\mathrm{p}<0.01$ compared with PBS; D) Transmission electron microscopy micrograph of PAPD polyplexes in PBS showing a rod-like structure, scale bar represents $200 \mathrm{~nm}$; Zeta potential of PAPD/siRNA complexes prepared at an N/P of 40 after incubation with PBS (E) and $\mathrm{N}_{2}$-bubbled PBS containing microsomes (F). 

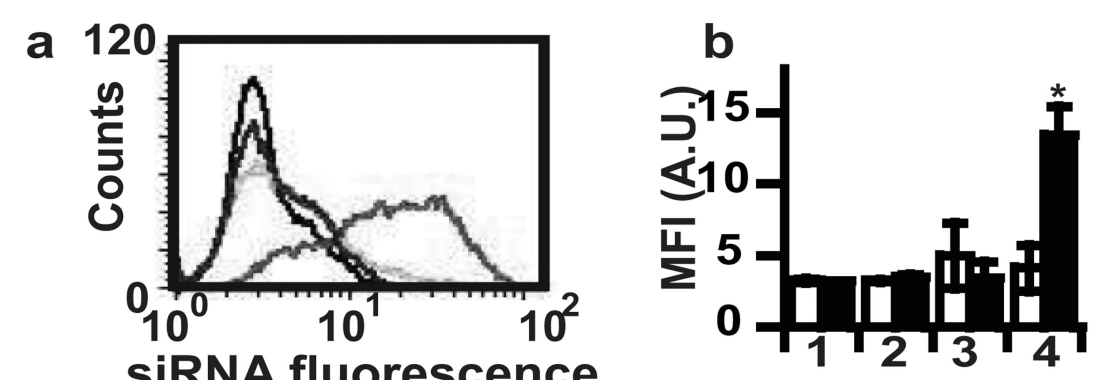

\section{C}
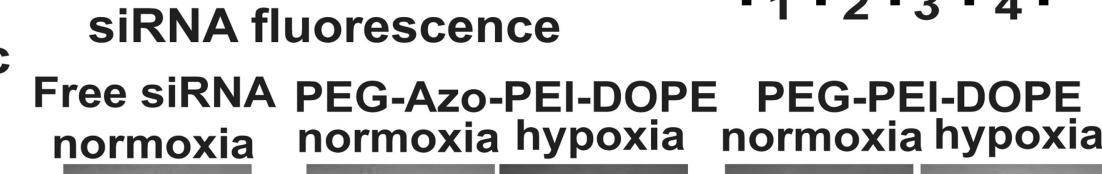

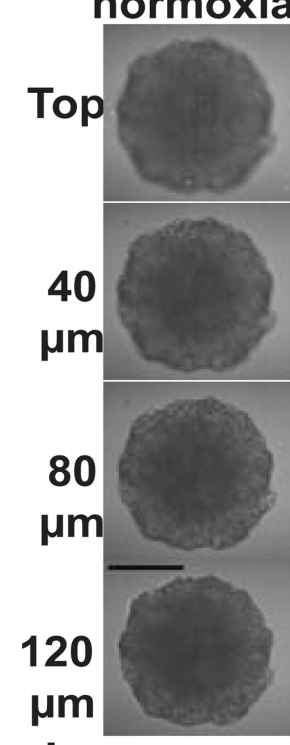

d

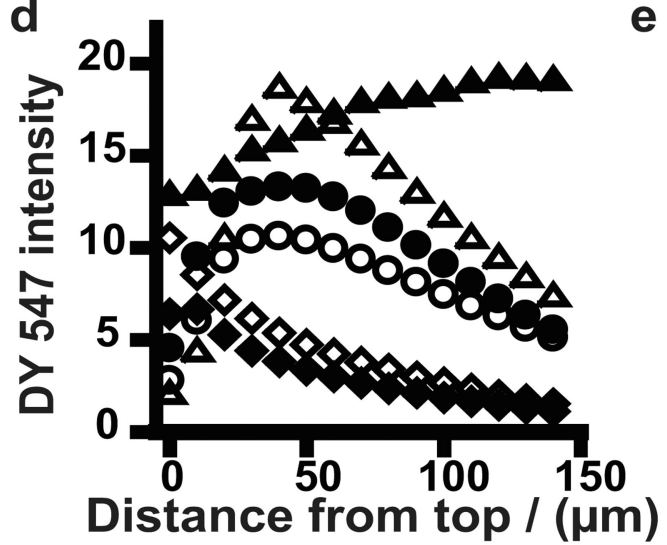

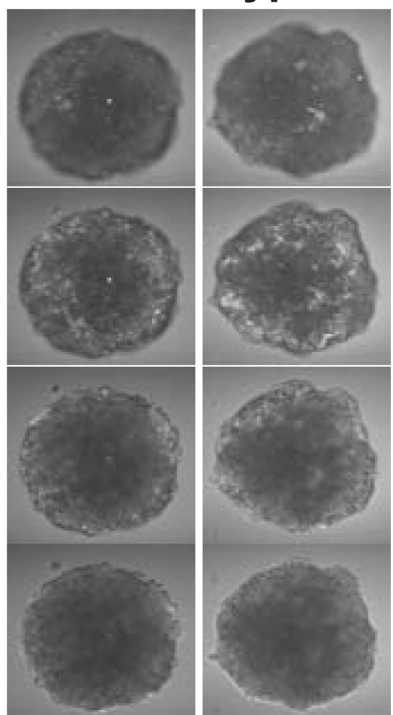
$120 \mu \mathrm{m}$ under hypoxia

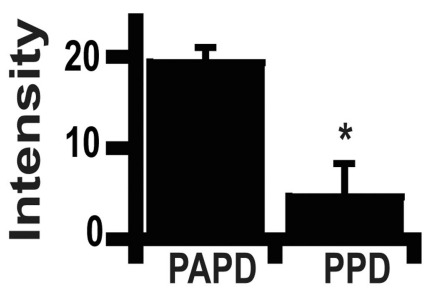

Figure 3. Internalization of siRNA in monolayers and distribution in spheroids Monolayers: A) Representative histogram plots of cellular internalization under hypoxia in the presence of $10 \%$ FBS. 1, PBS-treated cells; 2, free FAM-siRNA; 3, PEG-PEI-DOPE/ siRNA; 4, PEG-Azo-PEI-DOPE/siRNA; B) Geometric mean of fluorescence of A549 cells incubated $24 \mathrm{~h}$ with the same formulations as in A under normoxia (white bars) and hypoxia (black bars). Spheroids: NCI-ADR-RES spheroids were incubated 4 h under normoxia and hypoxia with DY 547-labeled siRNA; C) Imaging of siRNA distribution by confocal microscopy. Scale bar represents $250 \mu \mathrm{m}$; D) Quantitation of DY 547 fluorescence from the 
spheroid surface after incubation with free siRNA ( $\diamond$ for normoxia, $\diamond$ for hypoxia), PEGAzo-PEI-DOPE/siRNA ( $\triangle$ for normoxia, $\Delta$ for hypoxia) and PEG-PEI-DOPE/siRNA ( $\bigcirc$ for normoxia, for hypoxia); E) Average intensity of fluorescence at $120 \mu \mathrm{m}$ from spheroid surface after treatment with PEG-Azo-PEI-DOPE/siRNA (PAPD) and PEG-PEI-DOPE/ siRNA (PPD) under hypoxia, * $\mathrm{p}<0.05$ compared with PAPD/DY 547 siRNA complexes. 


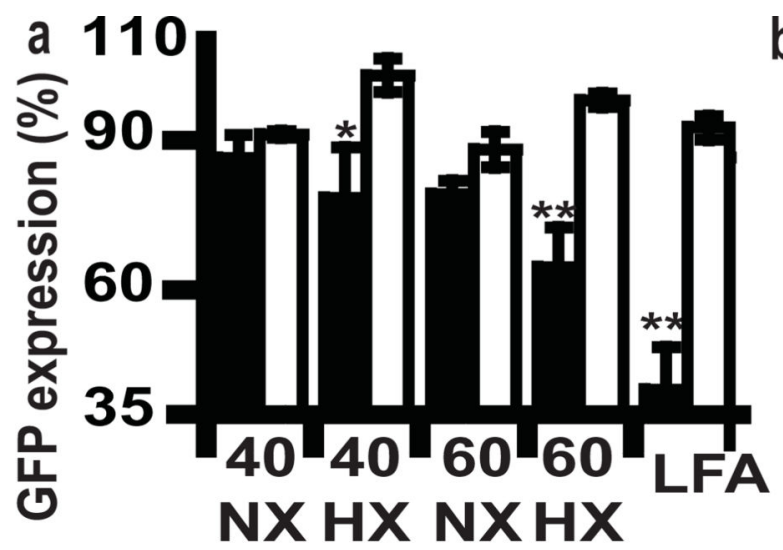

b

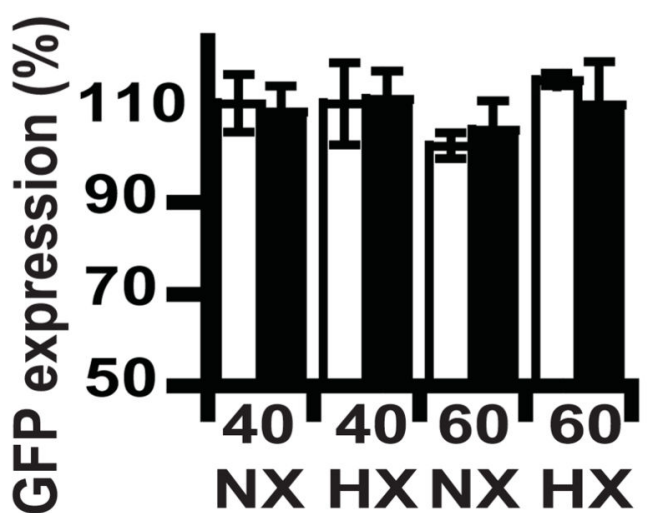

C

d

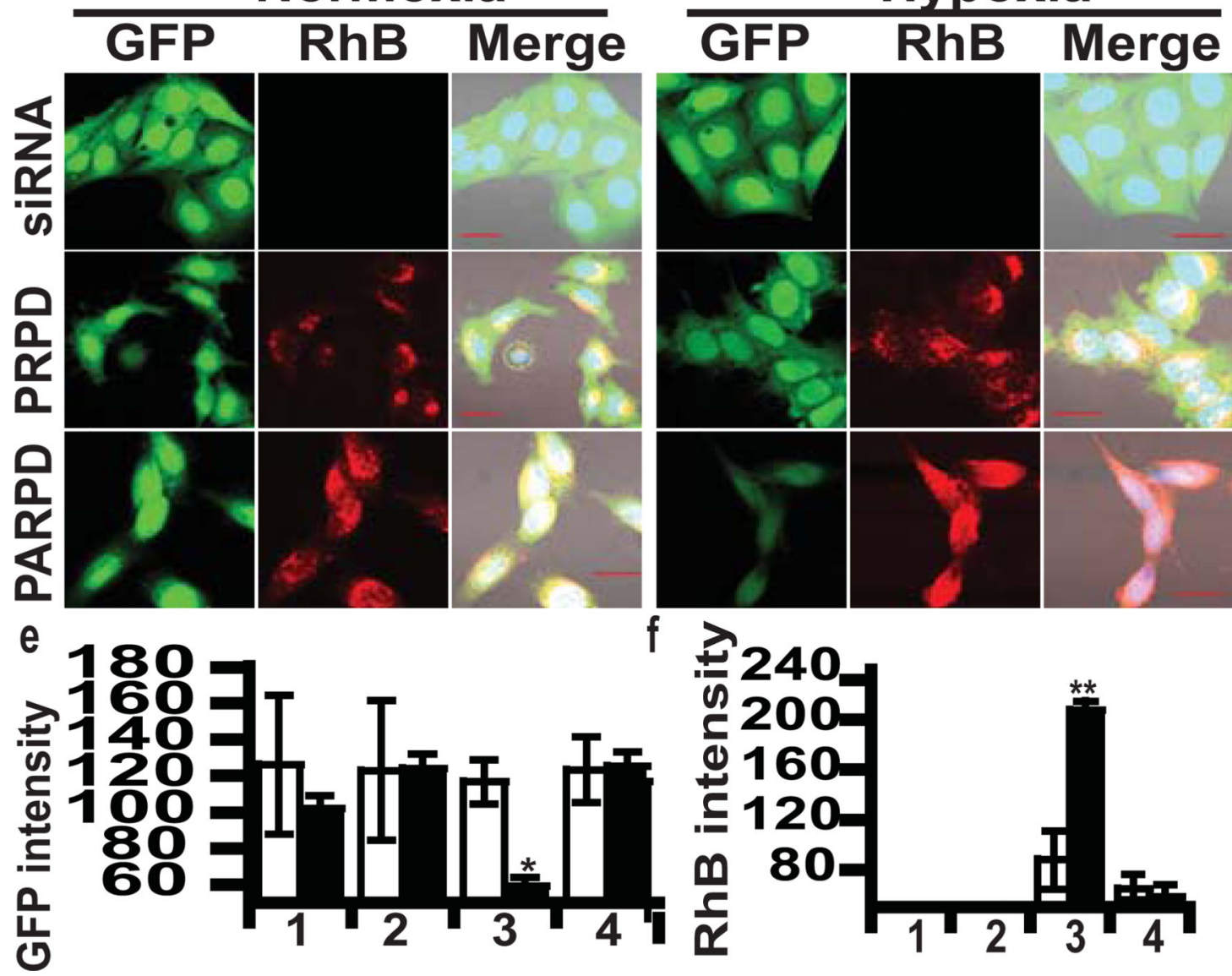

Figure 4. GFP down-regulation in vitro. GFP down-regulation was evaluated both by flow cytometry $(\mathrm{A}, \mathrm{B})$ and microscopy $(\mathrm{C}-\mathrm{F})$ after transfection under normoxic $(\mathrm{NX})$ or hypoxic $(\mathrm{HX})$ conditions

Relative geometric mean fluorescence from FACS analysis of HeLa/GFP cells transfected with (A) PEG-Azo-PEI-DOPE (PAPD)/siRNA complexes, (B) PEG-PEI-DOPE (PPD)/ siRNA complexes in the presence of $10 \%$ FBS. Polyplexes were prepared at N/P ratios of 40 and 60 with anti-GFP siRNA (black bars) or scramble siRNA (white bars). Lipofectamine 2000 (LFA) was used as a positive control, * $\mathrm{p}<0.05$, ** $\mathrm{p}<0.01$ compared with scramble siRNA complexes. CLSM images of HeLa/GFP cells transfected with rhodamine B-labeled 
copolymers PEG-Azo-Rhodamine-PEI-DOPE (PARPD), PEG-Rhodamine-PEI-DOPE (PRPD) and GFP siRNA under normoxia (C) and hypoxia (D). Mean pixel intensities of GFP (E) and rhodamine B (F) after transfection under normoxia (white bars) and hypoxia (black bars); 1: PBS, 2: free siRNA, 3: PARPD, 4: PRPD. $\mathrm{p}<0.05$, ** $\mathrm{p}<0.01$ compared with normoxia. 

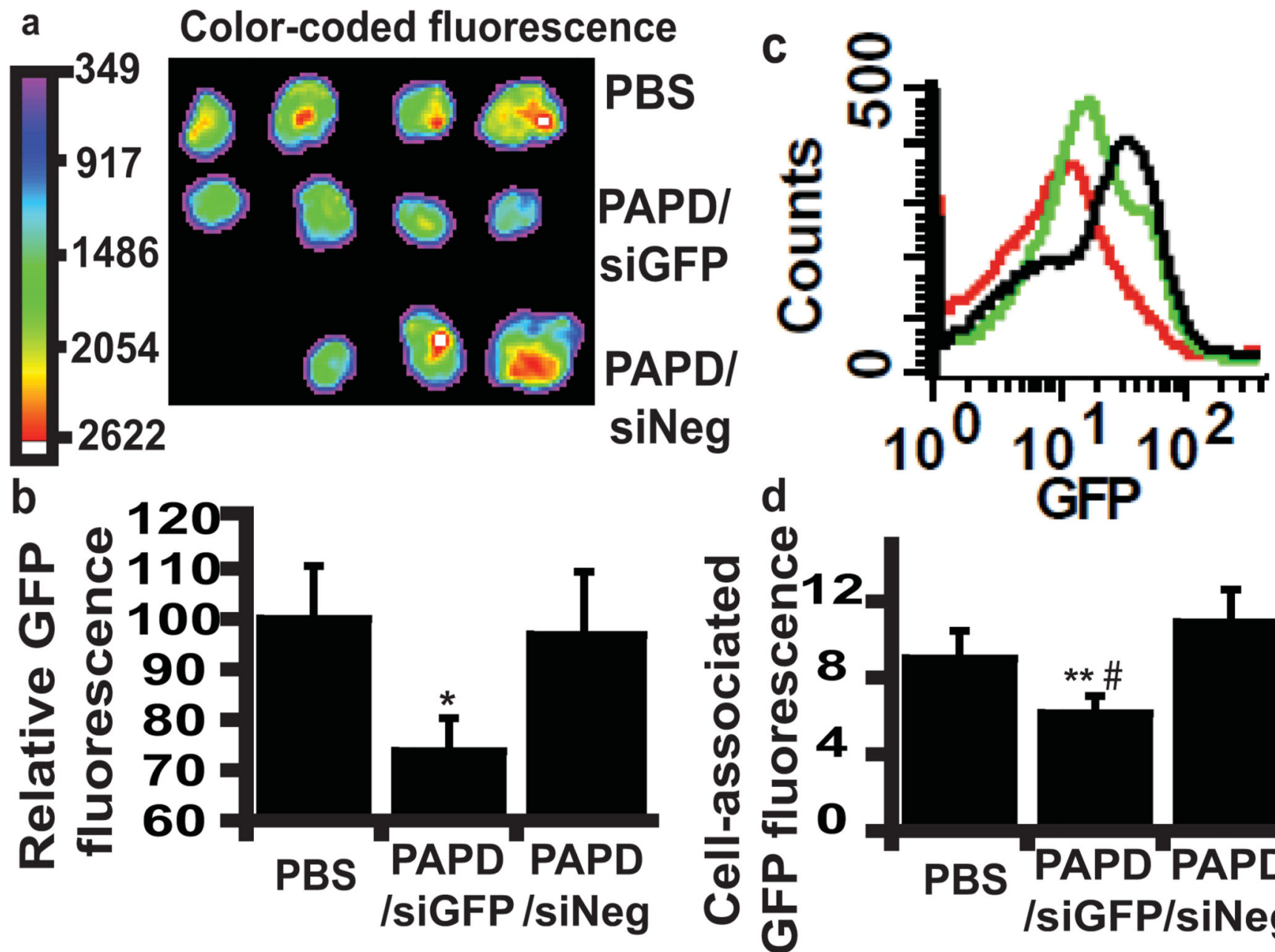

Figure 5. In vivo silencing activity

A) Ex vivo fluorescence optical imaging of tumors $48 \mathrm{~h}$ after intravenous injection of PBS $(\mathrm{n}=4)$, PEG-Azo-PEI-DOPE/anti-GFP siRNA complexes prepared with (PAPD/siGFP, $\mathrm{n}=4$ ), PEG-Azo-PEI-DOPE/negative siRNA complexes (PAPD/siNeg, $n=3$ ) at a dose of 1.5 $\mathrm{mg} / \mathrm{kg}$ of siRNA in $200 \mu \mathrm{L}$ PBS. B) Relative GFP fluorescence from tumors. Student's t test was performed, *p<0.05 compared to PBS or PAPD/siNeg; C) Cell-associated fluorescence of dissociated tumors by flow cytometry and representative histogram (D). Green, PBS;

Red, PAPD/siGFP; Black, PAPD/siNeg. Right, cell associated fluorescence from dissociated tumors. Only PAPD/siGFP led to a significant decrease of GFP expression (Student's t test, $* * \mathrm{p}<0.01$ compared to PBS, \#p<0.001 compared to PAPD/siNeg). 